\title{
Study on the Solar Tracking Control System Based on the Single Chip Microcomputer
}

\author{
Shihong Wu, Lulan Zhang \\ College of Information and Electrical Engineering, Shenyang Agricultural University \\ 120 Dongling Road, Shenyang City, China \\ wushihong8888@syau.edu.cn; 1294481710 @qq.com
}

\begin{abstract}
In order to solve the problem of low efficiency of solar energy utilization in the process of solar power generation, a solar tracking control system is designed on the basis of the single chip microcomputer. Its basic principle is using single chip microcomputer to control the rotation of the motor to track the sun. The system can automatically adjust the angle of sunlight receivers with sunlight, in this way, the sunlight receiving device can keep the vertical with the sun's rays, which realized the sun tracking and improved the efficiency of collecting the solar energy. The test results show that the solar energy receiving rate increased about $32 \%$, the purpose of solar tracking control system is achieved. On one hand this system provides a reference for all kinds of solar energy receiver, on the other hand, it will promote the efficiency of solar energy receiving.
\end{abstract}

Keywords: Solar energy; Single chip microcomputer; Solar tracking control system

\section{Introduction}

At present, the non-renewable energy like the coal and oil is widely used as the rapid development of the society. Considering the properties of non-renewable fossil fuels and the severe weather such as fog caused by the solid particles in the combustion process, the development and utilization of clean and renewable energy is a hot topic nowadays, especially for the solar energy.

According to the different methods of solar heat collection, solar thermal power generation system is classified into parabolic trough type, dish type, tower type, solar chimney type, etc. [1] For the decades of exploration and research, people have reached a consensus that at present only parabolic trough type has achieved commercial operation, meanwhile is the most mature technology of solar thermal power generation [2]. In the early 1990s, there were nine parabolic trough solar thermal power generators with installed capacity 354MW run formally, the annual output can reach 10TW.h in California of the United States. Spain has developed a new generation of parabolic concentrator to reduce the cost of power generation and improve the efficiency of power generation, funded by EU Framework Project for Research since 1998[3].

In recent years, some researches on solar power generation have been done in China. In the 1970s, a lot of basic experiments on solar energy were done in some scientific research institutions in China, and a $1 \mathrm{KW}$ solar thermal power generation simulation system was built [4]. Seven years ago, first 70KW tower solar thermal power generation system which was presided over by academician Zhang yaoming in China passed the appraisal and acceptance .Based on these researches Guangzhou institute of Chinese academy of sciences also took the further research and exploration on the parabolic trough solar thermal power generation system and achieved some important achievements.

\section{Overall Design Ideas}

In this paper, the method of Angle tracking is applied to intelligent control photovoltaic panels Angle, the first thing is to set the photoelectric checking device to judge whether it is day or night. During the daytime, a series of procedures set in the day begin to run; at night, interrupt program will be in response to reduce the energy consumption of the system which is in standby state of dormancy and waiting for the arrival of the next day. Secondly, during the day the intensity of sunlight is judged again to judge whether it is strong sunlight sunny weather or overcast weather with lower sunshine intensity to control the system in a state of dormancy or at work. Finally, the front information is processed to drive 
mechanical parts ultimately, namely, two stepper motors' rotation turns the sunlight collection device to realize real-time tracking of sunlight.

\section{Hardwares}

\subsection{Single Chip Microcomputer}

Type AT89C51 single chip microcomputer is taken in this system. The design idea of AT89C51 is static logic, its operating frequency can be as low as $0 \mathrm{~Hz}$, meanwhile it has two kinds of modes to save power. One is Idle Mode, in which the CPU stops working, but the timer/counter, RAM, interrupt system and serial port continues to work. The other mode is Power Down Mode, in which the on-chip oscillator, timer and all functions stop working, except that the data which the on chip RAM saved retain until the hardware resets next time. There are 40 pins in AT89C51, which are divided into doublein-line Package (DIP) and square package. The pins are shown in figure1.

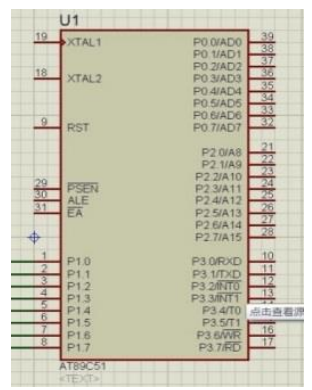

Fig. 1: AT89C51 microcontroller pins.

\subsection{Photoelectric Detection Circuit Design}

In order to avoid unnecessary energy wasting, it should be determined that whether it is sunny day when using the method of Angle tracking. The circuit diagram is shown in figure 2.

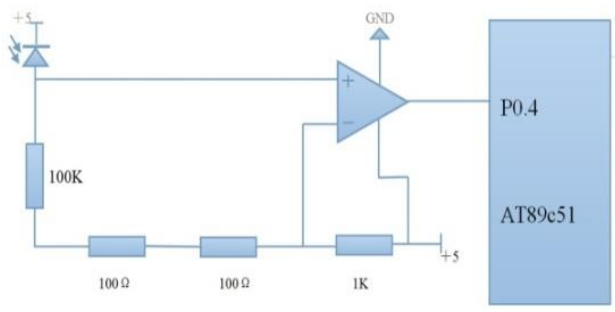

Fig. 2: Sunlight intensity detection.

The 2CU101D type photosensitive diode is adopted as a photosensitive element in this circuit to judge the strength of the sun. In the comparison circuit, a set of operational amplifier circuits are adopted, which the output of the op-amp connects microcontroller P0.4. AL0 inverting input terminal voltage, AL0 in-phase input terminal photodiode anode. Through the experiments the following resistors $\mathrm{R} 0=100 \mathrm{k} \Omega, \mathrm{R} 1=100 \Omega, \mathrm{R} 2=100 \Omega, \mathrm{R} 3=1 \mathrm{k} \Omega$ are determined 。

When it is cloudy, it is unable to conduct the photosensitive diode in the circuit because of the characteristics of the photodiode in a weak sunshine. These signals which cannot conduct make the op-amp output a low level, via operation and amplifier, at the moment P0.4 of the single chip microcomputer will get a low level, and then the system is controlled into dormancy by subsequent circuit and programs.

When the weather is sunny, the strength of the sun is enough to make the circuit of the photosensitive diode conduct, as the electric potential of the op-amp in-phase input is higher than the inverting input at this moment, a set of comparison circuit can be obtained, so the P0.4 of this single chip microcomputer can be detected by a high level, which is put from the output of the op-amp, and then the program can continue to run, so is the Angle tracking by the subsequent circuit and program control. 


\subsection{Motor Control Circuit}

The rotation of the motor is controlled by a single chip microcomputer. By the detection circuit mentioned above, the light signal is converted, step by step, into the electrical signal that can be identified by the single chip microcomputer. Based on these signals, the single chip microcomputer can control the system to achieve the effect of sun tracking. Based on controlling the conduction or deadline of the two levels of NPN transistors, the single chip microcomputer can control the relay closed or disconnected, thus to achieve controlling motor rotation. The control circuit as shown in figure 3 .

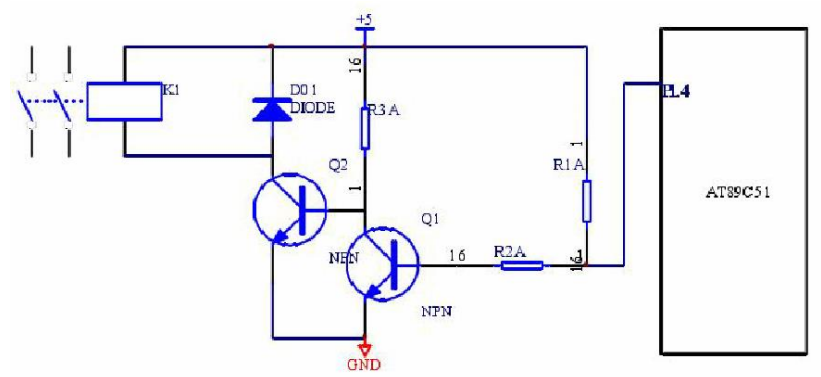

Fig. 3: motor control circuit.

Complete control circuit is composed by four groups of circuit with the same as above, which is a quarter of the whole control circuit. The four branches are respectively output by the P1.4 - P1.7 port of the AT89C51, as each group is connected by a relay, every two groups control a stepper motor, so that the forward and reverse rotation of the machine can be achieved.

Its working principle is that: When photosensitive diode is under the sun, the light signals are converted into the electrical signals which the AT89C51 single chip microcomputer can identify through a series of conversions by the front light detection circuit, namely the single chip microcomputer P2.0 will get a low level. The system program will put P1.4 reset, so the first transistor of the control circuit is turned off, the second transistor is turned on, and then the relay is closed, motor gets the current to rotate .In this way, the control of the motor by single chip microcomputer is realized.

\subsection{Clock Circuit Design}

Time should be counted and controlled when the Angle is tracking. As the use of single chip microcomputer's local clock cycles to the count in the process of the long time Angle of tracking, there would be an accumulative error that causes the system failure, so we should use professional clock chip for timing.

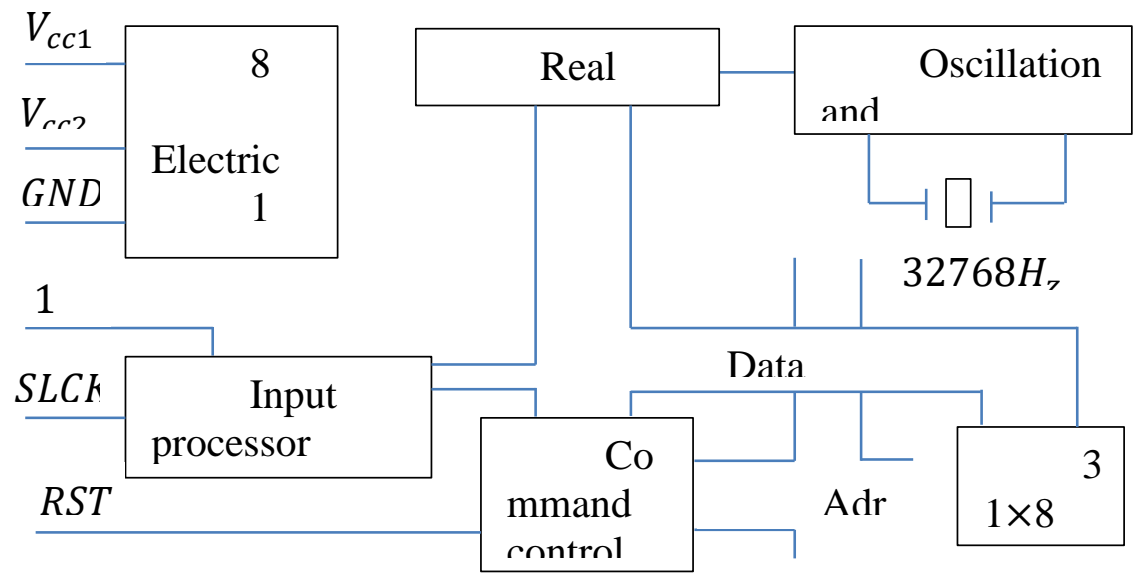

Fig. 4: DS1302 serial clock chip. 
The serial real-time clock chip DS1302 that produced by DALLAS company is adopted in this system. This chip is smaller, the attachment is simple, only has a $32.768 \mathrm{~Hz}$ crystal oscillator, has good anti-interference, and write protection, with $31 \times 8$ ram memory for storing data. When DS1302 chip is connected to single chip microcomputer, three lines including the I/O clock SCLK, cable line and reset RST should be connected .Data stream is transferred with a 1-31 bytes at a time[5]. The schematic of serial clock chip is shown in fig. 4.

The connection of the DS1302 and single chip microcomputer as shown in fig. 5.

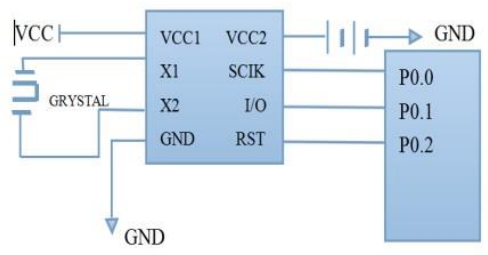

Fig. 5: Clock chip connection circuit.

RST pin has two functions: one is that the RST can transmit by single-byte or multi-byte data; the other is the RST control logic which allows the address/command sequence into the shift register [6].

If RST is low-level, then I/O pins become high impedance states, the data transmission shut off. Where Vcc $>2.5 \mathrm{~V}$, RST is logic 0 , only if the RST changes logic 0 to logic 1 , SCLK is logic 0 . The clock SCLK is rising edge carried on the clock sequence of falling edge. When the data is input, the front of the clock rising edge is effective, and the data output behind the clock falling edge [7].

\section{Software Design}

\subsection{The Whole Program Flow}

The running order of the whole program is as follows: first of all, judgment of day or night, system starts interrupt program at the night, then the system enters into a dormant state. Otherwise, the system comes into Angle tracking state. System operation flow as shown in fig. 6.

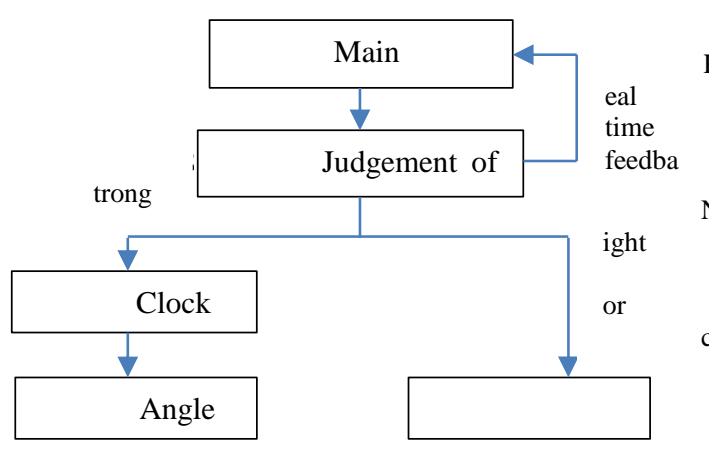

Fig. 6: Execution Flow of the System.

\subsection{Design of Angle Tracking Module}

In this system, cloudiness or sunniness is judged by the value of electric potential of the single chip pins P0.4. It is programmed that Angle tracking program runs when P0.4 get low level. After the start of the Angle tracking, firstly read the time of the current time clock chip, by using the default formula, and then calculate the solar altitude and azimuth direction height respectively, at the current position of the solar energy collection, secondly, calculate the height again after 15 minutes, and calculate the difference between the two times, then the time of the motor rotation can be calculated by the height difference of the two times. The program flow chart of the sun Angle tracking mode is shown in fig.7. 


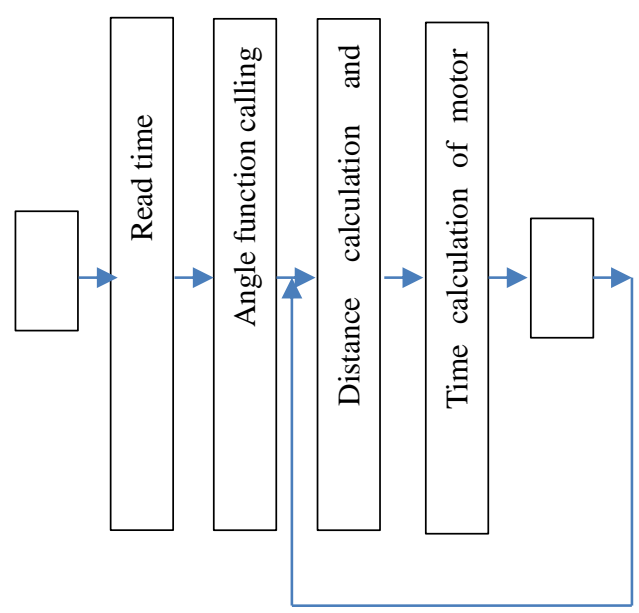

Fig. 7: Control flow.

\subsection{The Mathematical Principle of Angle Tracking}

The sun's movement rule is changing in years (365 days), the solar altitude $(\theta \mathrm{h})$ and solar azimuth $(\theta p)$ are constantly changing in every fixed region every day. Only to find out the relationship between the solar altitude or solar azimuth and time in a fixed region, and write a programs and put them into the single chip microcomputer to control the sunlight collection plane always keeping vertical with the sun's rays. Thus it can greatly improve the efficiency of the solar collection, and generate much electricity.

By using the experience of declination and date in astronomy, suppose a corresponding interval of the 365 days in a year is $[0-\pi]$, sun angle: $\theta 0=2 \pi(\mathrm{dn}-1) / 365$, where $\mathrm{dn}$ represents the date, $\mathrm{n}$ represents number of days, let January 1 st is equal to 1 and December 31 is equal to 365 , from 1 to 365 days represent a year

Declination angle:

$$
\begin{gathered}
\delta=0.00689-0.39951 \cos \theta_{0}+0.07208 \sin \theta_{0} \times 0.0068 \cos 2 \theta_{0} \\
+0.0009 \times \sin 2 \theta_{0}-0.00269 \cos 2 \theta_{0}-0.0029 \cos 3 \theta_{0}+0.00151 \sin 3 \theta_{0} \\
\text { solar hour angle: } \omega_{0}=\text { solar hour } \times 15-180
\end{gathered}
$$

The unit of $\omega$ is degree, namely the sun turns 15 degrees per hour

$$
\text { solar hour }=\text { Beijing hour }+(\text { correct degree } 120) \times 4 / 60+\text { time difference }
$$

time difference

$$
=0.000076+0.001869 \cos \theta_{0}-0.032076 \sin \theta_{0}-0.01416 \cos 2 \theta_{0}-0.04085 \sin 2 \theta_{0}
$$

The unit of time difference is hour,time difference $=$ time difference(radian) $\times 12 / \pi$

Let $\theta_{h}$ as the sun altitude angle at this time, $\theta_{p}$ as the sun azimuth angle, $\psi$ as the local geographic latitude Thus:

$$
\begin{gathered}
\sin \theta_{h}=\sin \Psi \sin \delta+\cos \Psi \cos \delta \cos \omega \\
\sin \theta_{p}=\cos \delta \sin \Psi / \cos \theta_{h} \\
\cos \theta_{p}=\left(\sin \theta_{h} \sin \Psi-\sin \delta\right) / \cos \theta_{h} \cos \Psi
\end{gathered}
$$


Using the three above angle functions to find out the relationship between the sun altitude angle $\theta_{h}$ and azimuth angle $\theta_{p}$ varied with time, at a latitude for $\Psi$ site in a casual, then a program is compiled and input the single chip microcomputer.

\subsection{Mathematical Model Building}

Mathematical model is as shown in fig. 8 .

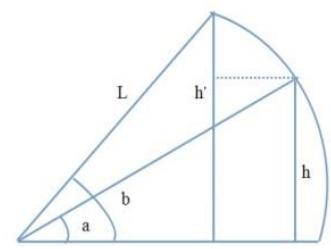

Fig. 8: Mathematical model.

Let the length of heat collection device is $2 \mathrm{~L}$. The $\mathrm{L}$ in this model diagram is half of the length of the heat collection device, is also the distance from the central spherical hinge of the fixed device to the around, rising height of the heat collection device is adjusted to $\mathrm{h}$ first time, at the moment ,the height of the sun is a ,the second adjustment of net rising height is $\left(h^{\prime}-h\right)$ ), at the same time ,the height of the sun is b.

Geometric relationship: $\quad h=L \sin a ; \quad h^{\prime}=L \sin b$

The second rising height calculated from above formulas:

$$
\begin{aligned}
& h^{\prime}-h=L(\sin b-\sin a) \\
& h^{\prime}-h=L(\sin b-\sin a)
\end{aligned}
$$

Let the transmission ratio of the ratio of worm as $i$, speed is $n 1$, then the speed of worm gear $n_{2}=n_{1} / i$, let the pitch of the screw as $d$, then the screw in a unit time, namely a pitch for a certain number of revolutions, its changing height $: \Delta_{h}=n_{1} \times d / i$

Let the first adjust time as $t$ gives :

$$
\begin{gathered}
h=L \sin a=\Delta_{h} t=\left(n_{1} \times d \times t\right) / i \\
t(a)=L \sin a \times i / n \times d
\end{gathered}
$$

In practical application, $L 、 d 、 i$ and $n_{1}$ are fixed, only a need to be read after the subprogram calculation. Therefore, when the first adjustment is done, the time of motor's rotation is achieved just find out the value of a. Similarly, the second adjustment function as:

$$
t(b)=L \times(\sin b-\sin a) \times i / n_{1} \times d
$$

The calculation above is to use the angle of the sun's height to represent the rotation time needed by motor tracking .In this way, the rotating time of the motor can be determined through the reading of time of internal designed function and clock chip. 
Table 4.1: Angle tracking data.

\begin{tabular}{|l|l|l|l|l|}
\hline \multirow{2}{*}{} & \multicolumn{2}{|l|}{} & \multicolumn{2}{l|}{$\begin{array}{l}\text { Angle of the sun } \\
\text { azimuth }\end{array}$} \\
\cline { 2 - 5 } time & Calendar & & $\begin{array}{l}\text { System } \\
\text { calculati } \\
\text { on }\end{array}$ \\
\hline & & & Calendar & \\
9:00 & 36.4621 & 36.3247 & -56.3832 & -56.2343 \\
\hline & & & & \\
$10: 00$ & 45.0636 & 45.4438 & -40.1765 & -40.4332 \\
\hline & & & & \\
$11: 00$ & 50.4034 & 50.4532 & -19.5544 & -19.3346 \\
\hline & & & & \\
$12: 00$ & 53.1934 & 52.3634 & -7.3243 & -7.3221 \\
\hline & & & & \\
\hline $13: 00$ & 48.4519 & 48.5654 & 18.5645 & 18.1512 \\
\hline & & & & \\
\hline $14: 00$ & 42.3843 & 42.0231 & 46.1952 & 46.1453 \\
\hline & & & & \\
\hline $15: 00$ & 33.2431 & 33.4745 & 61.4832 & 61.3142 \\
\hline
\end{tabular}

Table 4.2: solar radiation energy data.

\begin{tabular}{|c|c|c|c|}
\hline & $\begin{array}{l}\text { Data } \\
\text { collecting } \\
\text { time }\end{array}$ & $\begin{array}{l}\text { Singlw axis } \\
\text { system }\end{array}$ & $\begin{array}{l}\text { Dual axis } \\
\text { syste }\end{array}$ \\
\hline \multirow{7}{*}{$\begin{array}{l}\text { Radiation } \\
\text { intensity of } \\
\text { the sun in } \\
\text { differed } \\
\text { period } \\
\text { unit } \\
\mathrm{MJ} / \mathrm{m}^{2}\end{array}$} & $9: 00$ & 223 & 398 \\
\hline & $10: 00$ & 331 & 522 \\
\hline & $11: 00$ & 442 & 582 \\
\hline & $12: 00$ & 500 & 612 \\
\hline & $13: 00$ & 542 & 641 \\
\hline & $14: 00$ & 572 & 708 \\
\hline & $15: 00$ & 548 & 621 \\
\hline \multirow[t]{2}{*}{$\mathrm{m}$} & $16: 00$ & 368 & 567 \\
\hline & average & 440.5 & 581.5 \\
\hline $\begin{array}{l}\text { Increasing } \\
\text { efficiency }\end{array}$ & \multicolumn{3}{|c|}{$(581.5-440.5) / 440.5 \approx 0.32$} \\
\hline
\end{tabular}




\section{Data Acquisition and Contrast}

The data calculated by the program single chip microcomputer of this tracking system is roughly the same as the Era Shuttle Calendar, as shown in table 4.1, in line with the allowable error range. It proves that the system runs stable and reliable, which has the practical. Collecting the solar radiation data of different time is as shown in table 4.2. The average collection heat of this system is $581.5 \mathrm{MJ}$ per square meter per hour, but the traditional system is $440.5 \mathrm{MJ}$. Year-on-year growth is around 32\%, which proves that the heat collection efficiency of this system is higher.

Data collecting location: Shenyang (Tokyo $123^{\circ} 25$ 'north latitude of $41^{\circ} 48^{\prime}$ ), the weather: sunny time: 9 - 16 test time: on March 31, 2016.

\section{Conclusion}

Compared to the traditional system which can't be real-time solar tracking, this system has the following advantages: (1)It is designed based on single chip microcomputer control system, and not only costs saving but also has a high stability. (2) By using the principle of the Angle tracking of astronomy, the theoretical foundation is accurate and reliable. (3)It can track the altitude angle and azimuth of the sun accurately and in real time, meanwhile has a high flexibility. Through the experimental data, the results show that, the heat collecting efficiency of solar heat collection surface controlled by this system increases about $32 \%$ than ordinary system. In a word, the solar collection efficiency of this solar tracking system is higher, which can get higher generating efficiency in solar power generation.

\section{Acknowledgements}

This work was sponsored by the Natural Science Foundation of Liaoning Province and the authors are extremely grateful to Science and Technology Department of Liaoning Province for funding this project under Grant Number 2014027001.

\section{References}

[1] W. Zekai, "Application and Development of the Photo-thermal Power Generation Technology by Using Solar Energy," Glass, vol. 6, pp. 39-41, 2012.

[2] L. Stoddard, J. Abiecunas, R. O’Connell, "Economic, Energy, and Environmental Benefits of Concentrating Solar Power in California," Kansas: National Renewable Energy Laboratory, 2006.

[3] L. Zhihui, L. Xinfeng, "State and Trend of Solar Parabolic Trough Power Generation Technology," Electrical Equipment, vol. 7, pp. 29-32, 2006.

[4] Z. Chunjiang, Y. Jinhuan, C. Zhonghua, Z. Qianling, "State \& Development of Photovoltaic Application," Energy Conservation Technology, vol. 25, pp. 461-465, 2007.

[5] Y. Defa, Z. Hongling, "How to Use the Trickle Charge Timekeeping Chip Ds1302," Information Technology and Informatization, vol. 1, pp. 92-94, 2006.

[6] Z. Zhendong, L. Yanbin, D. Xiaolong, J. Zhantao, "Serial Communication of Tms320F28335 and Clock Chip Ds130," New Product \&Tech, vol. 3, pp. 50-53, 2016.

[7] Z. Liwei, "Study on solar Follows the System Automatically," Jinlin University, 2007. 